\title{
Analytic and simulation modeling of plant-animal populations in Russian tundra
}

\author{
Trashcheev Rostislav, Boranbayev Askar ${ }^{2}$, Boranbayev Seilkhan $^{3}$, Sarancha Dmitry $^{4}$, \\ Lyulyakin Oleg ${ }^{4}$, Yurezanskaya Yulia ${ }^{4}$
}

${ }^{1}$ Institute of Fundamental Problems of Biology of the Russian Academy of Sciences, Pushchino, Russia

${ }^{2}$ Nazarbayev University, Astana, Republic of Kazakhstan

${ }^{3}$ L. N. Gumilyov Eurasian National University, Astana, Republic of Kazakhstan

${ }^{4}$ Dorodnitsyn Computing Centre of the Russian Academy of Sciences, Moscow, Russia

\section{Email address:}

tslav85@mail.ru (T. Rostislav), aboranbayev@nu.edu.kz (B. Askar.), sboranba@yandex.kz (B. Seilkhan), saran@ccas.ru (S. Dmitry), oleg217@yandex.ru (L. Oleg.), july@ccas.ru (Y. Yulia)

\section{To cite this article:}

Trashcheev Rostislav, Boranbayev Askar, Boranbayev Seilkhan, Sarancha Dmitry, Lyulyakin Oleg, Yurezanskaya Yulia. Analytic and Simulation Modeling of Plant-Animal Populations in Russian Tundra. Computational Biology and Bioinformatics.

Vol. 2, No. 3, 2014, pp. 43-51. doi: 10.11648/j.cbb.20140203.13

\begin{abstract}
This article describes a mathematical modeling method of an ecological biology system; this method uses computers. Hypotheses about the leading mechanisms of fluctuations for tundra animals population's number are formulated. An analysis of difference and differential equations and their manifestations in the community model "vegetation - lemmings - arctic foxes" and in an individual-oriented model of a lemming population are performed. This method uses research results including a full set of operations, namely from a substantiation of an object choice, a selection and processing of a biological information to the construction of a set of interconnected models. The given approach is used in the analysis of animal fluctuations by means of the tundra community models "vegetation - lemmings - arctic foxes", "vegetation - reindeer", and the individual-oriented model of the lemming population.
\end{abstract}

Keywords: Ecology of Biosystems, Tundra Populations, Chaos, System Dynamics

\section{Introduction}

The mathematical modeling method of an ecological biology system specifically features: basic universal equations for analysis of the natural ecological biology system are absent (the analogues of equations in physics); theoretical (mathematical) methods are lack and it is necessary to evaluate them by experimental researches; some functional characteristics of ecological objects are absent, they are estimated with a help of experts; many biological research results are uncertain.

General assumptions are not connected with detailed representations of biological characteristics. They are connected with the model choice. Besides, they are based on mathematical equations. The information filling the model is realized according to its structure. But is this choice successful?

A model inevitably simplifies the situation. Only the results of computational experiments with a full formed model may show the success of this choice.

An effective tool is necessary for creating the model in a lack of quantitative data, at conditions of constant readiness to review the model assumptions and its structure. First of all, the success of modeling depends on the efficiency of an interdisciplinary dialog.

The appearance of J. Forrester's system dynamics (see [1]) have made such interdisciplinary toolbox available; it has been based on the method of simulation models creating in a dialog with experts. This approach lets one take into account virtually all proposals of the experts in either quantitative or qualitative form. The relative simplicity of the resulting models lets perform a comparative analysis for different sets of original assumptions, data, and hypotheses.

However a large simulation model is not amenable to a parametric analysis.

The construction of many variants for the model and their analysis leads to the formation of mathematics, i.e., 
the "designer" representations of the object. This contributes to the joint selection of the model structure. A biological information lets accept various intuitive ideas for a consideration.

The main stimulus for the development of modeling technology is enclosed in resolving the contradiction between the possibility of a detailed description and a desire to avoid the threat of a "model's immensity".

The aim of this paper is to formulate the following. The simulation model ideally built (from biology and mathematics point of view) is a tool for the preliminary study of the object. A basic (detailed) simulation model should be a component for a set of interrelated models including simplified models.

The simplified models have less variables. Moreover, they allow a detailed analytic study, allow to estimate the object "as a whole", configure the initial simulation model for the necessary sub-models, and make the hypotheses about the leading mechanisms of the phenomenon under study.

To solve the aforementioned problems the method of complex studies (COST) is proposed. This method includes the entire sequence of operations: collection, filtering, analysis, and processing of an input (biological) information; the justification and the construction of simulation models, and the analysis of their properties; the formulation of a simulation system, i.e., a set of interrelated models on different itemized levels; the set includes the simplified models admitting the analytic (portrait) study; the formulation of hypotheses on the leading mechanisms for the phenomenon under consideration.

Pattern-oriented modeling is a general strategy for complex systems modeling. Many structures observed at different scales and hierarchical levels are used for model's optimization and calibration, in addition, for testing and choice of key processes for sub-models (see [2]). This approach is the closest to our COST method.

One can create the simplified (analytic) models with a joint analysis of the ecologo-biological information and the results of computational experiments based on reductions of basic simulation models. Why is the existence of the simplified model useful? How to use it? Given paper discusses this.

The complex research approach has been suggested in order to model the tundra community. Basing on expert estimates of relationships researchers have created the "vegetation - lemmings - arctic foxes" (VLF) simulation model such that it takes into account seasonal changes in parameters.

To understand better the mechanisms forming the dynamics of tundra animal populations the researchers have justified the use of one-dimensional difference equation as the simplified model relating the lemming population size (the leading unit in the VLF model) in two consecutive years.

This simplified model plays a special role in studying the population's number fluctuations for tundra animals. This has led us to search a closer connection between difference equation and the original simulation model VFL. Basing on the joint analysis of the ecologo-biological information and the results of the computational experiments we have been able to formulate and solve an inverse simulation problem (see [3]). This problem introduces the additional assumptions such that they let us get the formulas relating the original community model's parameters and the parameters of the difference equation. The VFL model is described briefly in the second section. The model's full description is performed in the paper [3].

The properties of the difference equation are considered in the third section. Nontrivial conclusions about these properties are made.

The simplified models and our previous modeling experience have led us to another level of description namely individual-oriented models (see $[4,5]$ ).

The modeling results don't depend on a specific parameterization, but they depend on the model's type. This is the important achievement of our researches.

The forth section describes the individual-oriented model of lemmings. The fifth section discusses the model "vegetation - reindeer".

\section{The Model "Vegetation - Lemmings - Arctic Foxes"}

In many ways despite the lack of study the tundra is an attractive object for the modeling. It is a relatively simple ecosystem with few species, the trophic relations are strained. It is very difficult for animals to survive there. To create a meaningful mathematical model we need some striking phenomenon. Our model will recreate this phenomenon.

The most popular "predator-prey" model was created to account for the fluctuations of the animal population's number. The main advantage of this object is the existence of the pronounced regular fluctuations. In particular the number of the arctic foxes and the number of lemmings are subjected to the fluctuations. The lemmings are the primary prey of the arctic foxes. The regular fluctuations give us the reliable testing effect in studying the population's dynamics. Regular peaks in the lemming populations were noted approximately once per three-four years (see [6]). The peaks were fixed once per three years on Taimyr peninsula.

At the construction of the model the following principles have been used: minimality, i.e., we use the simplest possible mathematical structure to simulate the phenomenon; systemness, i.e., we take into account the diversity of the relations within and outside the objects under study; compatibility, i.e., we use the assumptions such that they do not contradict available ecological data.

A biophysical analysis for the structure of the above-ground pasture part of the tundra biocoenosis has indicated the possibility to consider the VLF community separately. The biophysical analysis is described in detail in 
the papers $[5,7]$.

Simulation object selecting and a choice of the structure for its mathematical description are a compromise between mathematical and ecological requirements.

To describe a model a mathematical structure "ecological constructor" is proposed. This structure makes the model modification relatively simple. The implementation of this idea is based on a combination of J. Forrester's system dynamics and the hypothesis of V. Volterra-Kostitsyn, i.e., the possibility of using the ordinary differential equations to describe the objects of ecological systems (see $[5,7,8]$ ).

The dynamics of the tundra community biomass is described by the non-autonomous system:

$$
\begin{gathered}
\frac{d V}{d t}=f_{V}(V, L, \gamma), \frac{d L}{d t}=f_{L}(V, L, F, \gamma), \\
\frac{d F}{d t}=f_{F}(L, F, \gamma),
\end{gathered}
$$

where $F, L, V$ are the biomass (number) dynamics of the arctic foxes, the lemmings and the vegetation (their food), respectively, $\gamma$ is the vector of system parameters. For each trophic level $X$ we have $f_{X}=R_{X}-M_{X}-D_{X}$, where $R_{X}$ is a growth, $M_{X}$ is a natural extinction, $D_{X}$ is an alienation (hunting).

The biomass dynamics for each trophic level $X$ is defined by three additive components. They are a reproduction rate, the alienation and the natural extinction. Each component is formed as a product of a constant and the respective function including the function estimated by experts.

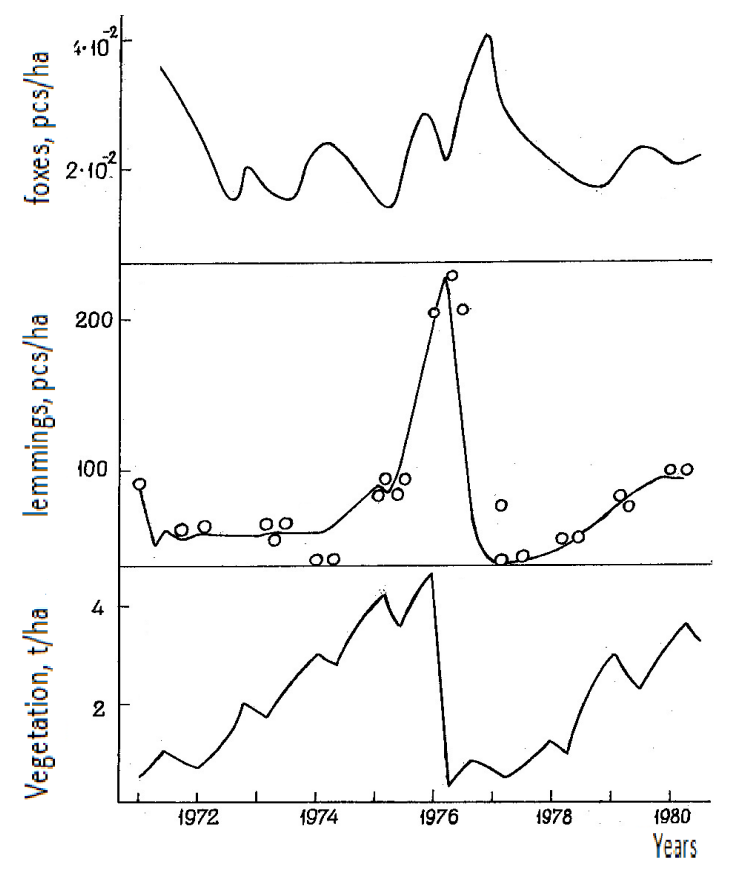

Figure 1. The results of the simulation experiment with the VLF model, the registered on Wrangel island (see [3]) trends of the hoof lemming are marked with the circles.

This approach corresponds to the level of our knowledge in the biophysics of ecological processes, the variety of assumptions, and lets us take into account different ecological hypotheses in different modifications of the model. We have created a large number of versions of this model. At first, we have used the idea of strong trophic interactions for the "predator-prey" kind literally. Then using the threshold dependence hypothesis (the rate of the lemming's biomass growth depends on the vegetation biomass (see [7])) and other hypotheses we have switched.

Basing on the expert data we collected we have constructed the first version of the model. It is a union of Forrester's and V. Volterra's approaches, also Volterra's "meeting hypotheses" have been emphasized (see [7]). This appears to be the main reason for the success of our modeling. This model was deconstructed when one of the species died, and soon afterwards the entire system died too The failures in the implementation have led us to search for alternative approaches and methods of a simplified description. The studies of zero isoclinic lines in the "vegetation-lemmings" system have led to the idea of using an analogy with a neural cell. This implies that we introduce in the second version of the model the threshold dependence of the lemming's biomass growth on the food availability. When a certain critical vegetation biomass is reached the lemming population's explosion happens and soon afterwards the food supply becomes depleted. We have controlled a rate of vegetation regeneration. It follows that this has let us control the model tuning. Thus we have proved a kind of the theorem of existence about the possibility of the reconstruction for the necessary dynamic modes with a model from the chosen class. Our usage of the "threshold model" has made possible to find in a computational experiment the relations between the parameters of the corresponding expert estimates and an average interval between the population peaks.

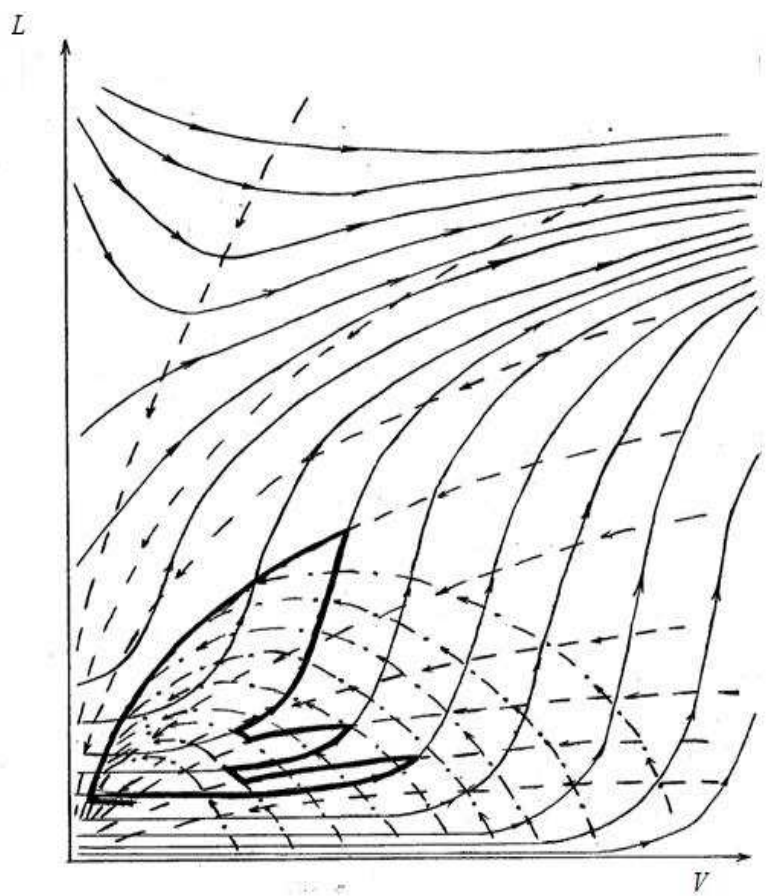

Figure 2. The phase portrait of the simplified subsystem "vegetation lemmings". 
The second version of the model has turned out to be unsatisfactory. Hence we have attempted to restructure the modeling process. There have been two ways of restructuring. Extending (deepening) of the biophysical knowledge about the biological properties of the biocenoses was the first way. Searching for efficient mathematical ways to express these properties was the second way. Having analyzed the results of computational experiments and ecological information we have understood the great importance of intrapopulation dynamics for the lemmings. The lemming population number's fluctuations affect all animals in the tundra community. We have introduced a new type of nonlinearity, namely the Allee principle (see [7]) such that it brings into the model the lemming density that is optimal for the reproduction. A large number of other modifying assumptions has been related to the increasing stability (trajectory "boundedness") of the model. Testing these assumptions we have used two biophysical criteria independently of expert estimates and axioms of classical models. Keeping the trajectories in the positive square was the first criterion. Reproducing of corresponding dynamic modes was the second one. The description for this version of the "vegetation-lemmings-arctic foxes" model is given in the papers $[5,7]$.

In computational experiments we have obtained threeand four-year cycles in lemming and arctic fox population's sizes fluctuations that are characteristic for tundra. Fig. 1 shows the results of one simulation experiment with the VLF model. The population dynamics of arctic lemming registered on Wrangel island (see [3]) is denoted by circles; Fig. 2 shows the phase portrait of the "vegetation-lemmings" subsystem constructed with numerical computations on the entire model for each season. Here the bold line represents one of the actually realized trajectories; thin lines designate phase curves in various seasons: dashed line, in winter (when lemmings do not reproduce); dot-and-dash line, in the nival reproduction period; solid, in summer (vegetation $V$ is along the horizontal axis, lemmings $L$ are along the vertical axis). As Fig. 2 clearly shows, during winter and spring the trajectories are attracted to the origin at the same time in summer the attractor is in the region of high lemming and vegetation density. Due to seasonal switching of the trajectories, fluctuations appear in the model.

There are a lot of modern papers relating a chaos and the dynamics of rodent's number, for example, see the paper [9].

The desire of deeper understanding the mechanisms of the population dynamics for tundra animals has led us to the closing stages of the COST method. The simplified model of a lemming population has been constructed (see [10]). Using a chaos phenomenon it determines the nature of the fluctuations. This model has a form of one-dimensional difference equation relating the number of the lemmings in two neighboring years. We have searched relationships between the simplified model and the initial simulation model. We have formulated and solved the inverse simulation problem, i.e., we have introduced the additional assumptions such that they give the formulas relating the parameters of original community model and the parameters of difference equation (see [3]).

To solve this problem the following simplifications have been realized. First of all, the arctic foxes have been excluded from the model as their influence on the lemming's population dynamics is negligible. Then we have realized a piece linearization such that it uses nonlinear relationships. The example of such linearization is shown in Fig. 3, where $g_{L}^{2}$ is a trophic lemmings function that formalizes the decline in the forages value in their deficit. This function is characterized with two constants (the first one is used in summer, the second one is used in spring). Thus the original system is reduced to a set of systems for two independent linear ordinary differential equations with constant coefficients (see [3]).

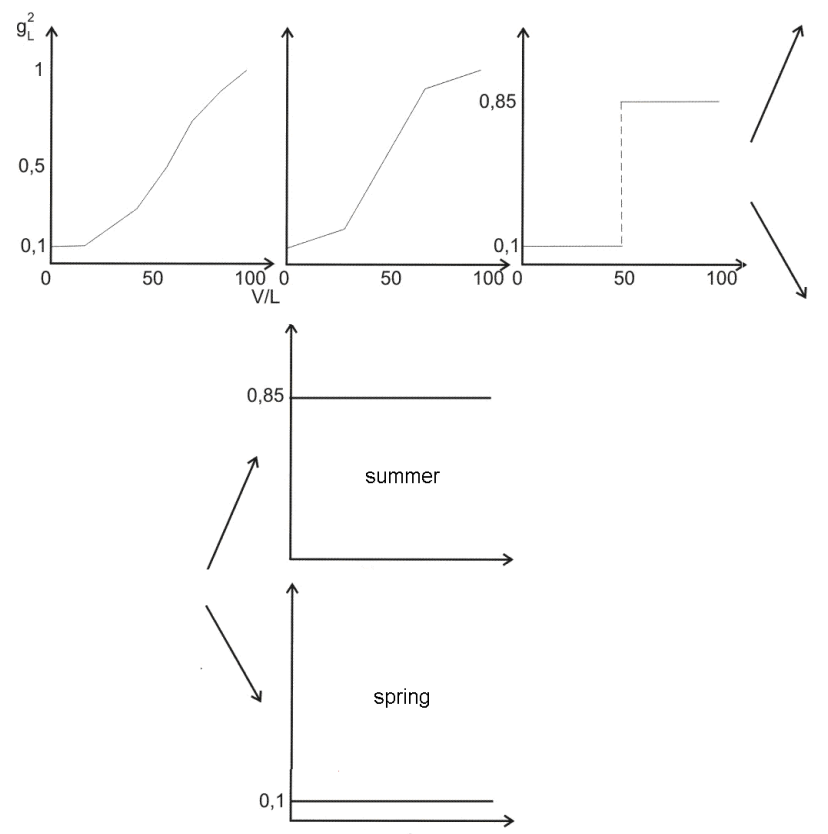

Figure 3. The successive stages of the trophic lemmings function's approximation.

The result is the difference equation that relates the number of lemmings in two adjacent years (see [5]). For the normalized variable $\widetilde{L}=L / L_{\max }$ it has the following form

$$
\widetilde{L}_{n+1}= \begin{cases}P \widetilde{L}_{n}, & \widetilde{L}_{n} \leq 1 / P, \\ 1-r\left(\widetilde{L}_{n}-1 / P\right), & 1 / P<\widetilde{L}_{n} \leq \widetilde{B}, \\ d, & \widetilde{L}_{n}>\widetilde{B} .\end{cases}
$$

Here $P$ is a growth of the lemmings biomass during the favorable year; the value $\widetilde{B}$ is determined from the conditions of a starvation in the late winter; $d$ is a normalized lemmings biomass in an optimal biotope (the notion of an optimal biotope was introduced in the paper [7] as a habitat with optimal conditions; in the optimal biotope 
a certain number of animals survives under any conditions); the coefficient $r$ characterizes the change in the lemmings biomass when there is not enough food in spring.

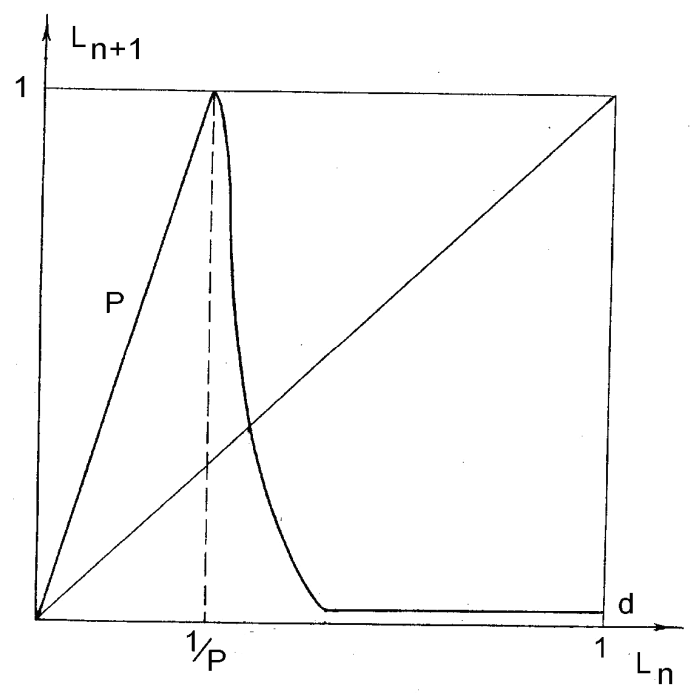

Figure 4. The graphical representation of the difference equation derived from the results of numerical experiments with the VLF model.

For comparison Fig. 4 shows a plot of the difference equation derived from the results of numerical experiments with the original VLF model, where $L_{n}$ designates a current year, $L_{n+1}$ is a next year, the value $1 / P$ determines the decrease of food below the critical level.

We have combined the models of different classes. The simplifications are not accurate as they are the source of linearization of expert functions but, nevertheless, allow a joint analysis of the models.

\section{The Difference Equation}

The simplified models in the form of difference (discrete) equations eliminate a nonlinearity in the species interaction for the VLF model. This fact has led us to fix the periodicity caused by the features of a seasonal model behavior

The analysis of the difference equation can justify the following hypothesis: the leading role in the fluctuations formation belongs to two dimensionless parameters. The first one is the growth of the lemmings biomass $P$. The second one is the proportion of survivors $d$ for the lemmings at the most adverse conditions. The conclusions obtained are in good agreement with one of the common hypothesizes: a combination of different factors forms the population fluctuation. In this case, these combinations are shown and their quantitative influence on the population dynamics is provided.

The computational experiments have been carried out with the changing parameter $d$ from 1 to 0 in (2) (see Fig. 5 ). We see the zones of stable cycles (white vertical sections) and the transition zones with more complex modes (black vertical sections). The stable zones are followed by the transition zones. The period of the stable zones changes in the sequence of natural numbers $(1,2,3$, $4, \ldots)$ from left to right.

The presence of the transition zones corresponds to a registered dynamics of real populations. When the clear three-year cycle is absent (at warmer regions compared to Taimyr) there are the two- and five-year intervals between the population peaks (see $[6,11,12])$.

The resulting difference equation can be a simple tool to predict the possible number of lemmings and foxes. However to assess the effects of anthropogenic influence we must use the full simulation model.

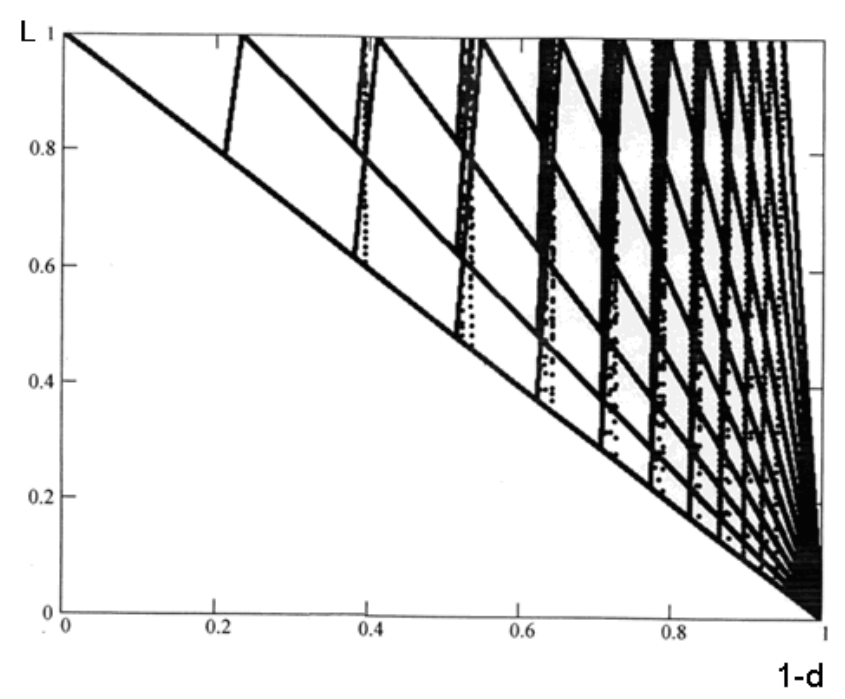

Figure 5. The results of the computational experiments on model (2): the dependence of trajectories on $1-d$. On the abscissa axis, the value $1-d$ is marked. The vertical section of the graph at a chosen value of $d$ consists of trajectory points.

\section{Individual-Oriented Models}

The simplified models in the form of the difference equations allow using the individual-oriented models to describe the population of lemmings (see $[4,13]$ ). The use of the individual-oriented models has led to a new level of working out in detail, i.e., we have taken into account ecological and physiological characteristics of individuals, especially their interaction (social mechanisms), the impact of their behavior on the environment (including the spatial features of the area), seasonal factors. The dynamics of the individual is determined by a set of behavioral rules such that they describe the individual interacting with the environment and / or other individuals.

A detailed description of the properties for individual-oriented models is given in the previous publications (see $[4,5]$ ). We present highlights.

The model year is divided into two periods: the breeding season (from February 1 to August 31) and the period of hibernation. The lemmings are described by their age, sex, viability potential, and stage of sexual development. The population changes are related to the animals movement.

After coming out of a hole the animal moves in a random direction. Encounters are possible when the lemming meets 
another one, the viability potential diminishing. Sometimes this potential reaches zero and the animal dies. The death also occurs when the maximum age is reached. When the animals of different sexes meet during the breeding period there is a certain probability of being pregnant for a female.

After some time descendants appear. They are at the parent hole about two weeks. The stage of sexual maturity occurs when the animals reach a certain age and find their own hole (the more detailed model is described in the papers $[13,14])$.

The computational experiments with individual-oriented models have allowed to produce the population fluctuations including the three-year period (see Fig. 6).

The graphic representation of the difference equation derived from the results of the computational experiments (the points on the graph) for the individual-oriented models is qualitatively close to the form of the difference equation obtained for the VLF model [5].

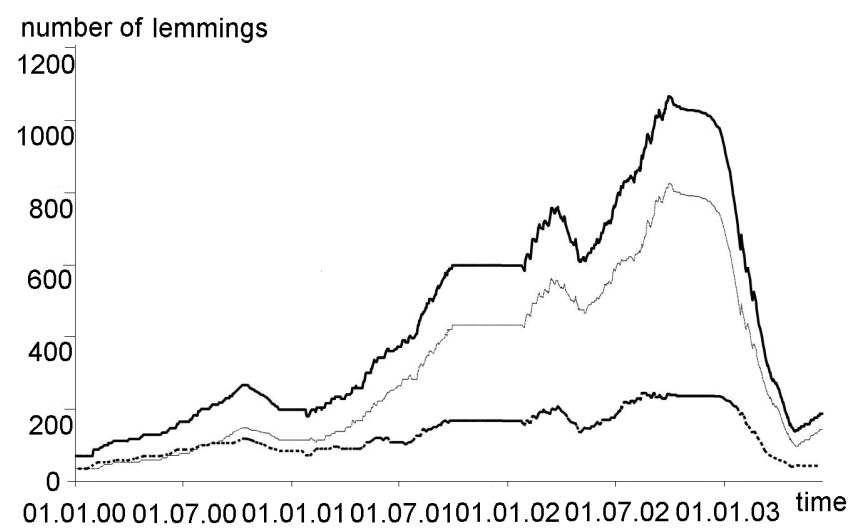

Figure 6. A typical three-year cycle (the thick line designates the number of the total population, the bold dash-and-dot line designates the number of individuals with increased resistance to encounters, the thin line designates an increased prolificacy)

The study of the individual-oriented models has been continued. The influence of the genotype of animals has been investigated. The population has been divided into the groups with different characteristics such as the increased prolificacy, the resistance to external conditions, etc.

Three versions of the model have been examined. The first version uses a random uniform distribution for different genotypes of the individuals. If the parents have a different genotype the child gets only one parent's features.

The situation of a genotype implementation has been considered in the second version. The first genotype has been introduced at initial distribution, the second one has been involved later. Further, the model parameters have been determined according to the results of computer simulation. The choice of parameters makes a simultaneous genotypes coexistence possible. As well as the range of parameters has been defined such that one genotype has been replaced by another there.

In the third case the descendants receive the averaged properties of both parents. In this case, after a while the population becomes genetically homogeneous. Also, the effect of food and dominant species has been studied.

We have succeeded in finding the parameters for stable three- and four-year cycles. Fig. 6 shows the typical three-year cycle. In our case, we see both genotypes survive.

\section{5. "Vegetation - Reindeer" Community Modeling}

We have extended the methodology of complex researches to other environmental objects. For this purpose we have chosen the reindeer population considered in the paper [15]. The reindeer population's dynamics was registered in Murmansk region (Lapland Reserve) during the period of 1929-1995 years [15].

Basing on the relationship between the population and feed resources the discrete mathematical model of non-exploited group of reindeers is considered in the paper [15]. This model takes into account the age structure of the animals.

Solving numerically the non-autonomous system of two ordinary differential equations of the first order built on the basis of complex studies method we have reproduced the similar dynamic modes without the age structure.

The data analysis (see Fig. 7) showed that the dynamics is cyclical with the oscillation period of $35-40$ years, the raises of reindeer number continuing 25-30 years. The population decreasing continues 10 years. The model [15] reproduces the dynamic conditions similar to the registered ones.

The similarity of the cyclical fluctuations in the "vegetation - lemmings - arctic foxes" model has led us to apply the complex research method. During the formation of the model we have also used three principles: minimality, systemness, compatibility. In addition, the hypothesis of critical levels of vegetation has been involved [16].

In the framework of these principles "vegetationreindeer" community has been allocated. In this case, it has been possible to use mathematical construction (2.1).

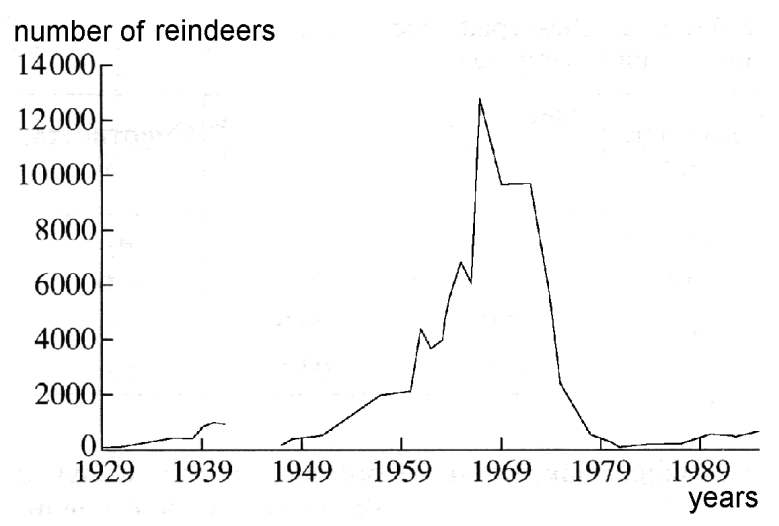

Figure 7. Experimental data. The registered reindeer dynamics in Murmansk region (Lapland Reserve) during 1929-1995 years [15]. The gap during 1942-1947 years is related to World War II when the deer meat was stacked for food. 


\subsection{Description of the Simulation Model}

In the original simulation model there are three modes: enough food (the increasing population), not enough food (the birth rate is equal to zero), the food is not available (high mortality, the reduction of population). In this model there is one expert function $(f d v)$ (see Fig. 8) formalizing the assumption of the critical vegetation levels [16]. Basing on the analysis of the numerical experiments with the simulation model we have introduced the additional assumptions simplifying the simulation model to the model that can be analytically studied. The analytical solution of the system of differential equations has a simple form when food is not available, but if there is a sufficient food supply the solution is expressed in terms of the Bessel functions [17]. The analytical solution can be a tool for configuring the simulation model's parameters.

The area of 100 sq.km. has been chosen for the modeling. The maximum number of the population in the given area is 120 animals. The maximum biomass of lichens is 10 centner/ha. The reindeer population's growth leads to the decrease in the biomass of lichens to 3 centner/ha. Then the growth is replaced by the population decrease. Thus the average biomass of the lichens 3 centner/ha is critical for this population, the food being not enough. The fecundity of the individuals drops to zero below 2.4 centner/ha of forage available and the mortality significantly increases. In accordance with the hypothesis of the critical vegetation levels [16], the diminishing of lichens reserves can not be complete and their recovery begins before the reindeer population reaches a minimum.

The initial simulation model has the form

$$
\left\{\begin{array}{l}
\frac{d V}{d t}=R_{V}-M_{V}-D_{V}, \\
\frac{d R}{d t}=R_{R}-M_{R},
\end{array}\right.
$$

where $V$ designates the the lichens biomass (tons/100 square $\mathrm{km}), R$ designates the reindeers number, the items of the system of the equations are calculated using the following formulas.

The vegetation growth $R_{V}=a_{1} \cdot\left(1-\frac{V}{V_{\max }}\right) \cdot V \cdot$ The natural death of vegetation $M_{V}=a_{2} \cdot V$. The vegetation alienation (grazing) $D_{V}=b_{1} \cdot R$ if the food is enough, if there is not enough food $D_{V}=V \cdot f d v(V)$. The increase of the reindeer population $R_{R}=D_{V} \cdot k p b r$ if the food is enough and $(V \geq \alpha) ; \quad R_{R}=0$ if the food is not enough and $(V<\alpha)$. The reindeer death $M_{R}=R \cdot b_{2}$ if the food is enough and $(V \geq \alpha \cdot 0.8) ; M_{R}=R \cdot b_{3}$ if the food is not available and $(V<\alpha \cdot 0.8)$.

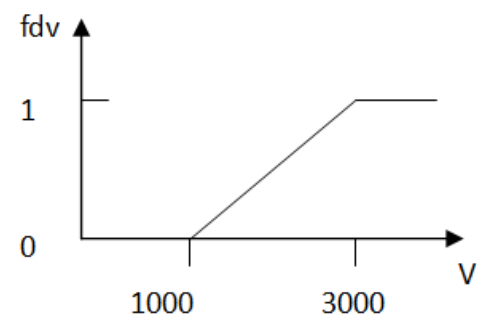

Figure 8. Function fdv. Decrease in value of food (V) during its deficit [16].

Consider the coefficients of system (5.1): $V_{\text {max }}$ designates the maximum of the lichens biomass, $a_{1}$ designates the coefficient of the vegetation growth, $a_{2}$ designates the coefficient of the vegetation extinction, $b_{1}$ designates the coefficient of the rate of the lichens consumption (tons per animal per year), $b_{2}$ designates the coefficient of the reindeer mortality if there is enough food, $b_{3}$ designates the coefficient of the reindeer mortality if there is not enough food, $k p b r$ designates the coefficient of the lichens biomass transferring to the reindeer biomass ( the coefficient of conversion), $f d v(V)$ designates the function formalizing the decline in the value of food during its deficit. Fig. 9 demonstrates the results of the computational experiments.

\subsection{Description of the Analytical Model of the Community}

There are 3 modes in the simulation model. There is enough food during the first mode, the population increase is observed. There is not enough food during the second mode, the birth rate is zero. The food is not available during the third mode, the high mortality and the population decrease are observed. In addition, there is one expert function $f d v$.

This model is found to be simplified as follows. We suppose the model to work in two modes. There is enough food and $V \geq \alpha$ during the first mode, the population increase is observed. The food is not available and $V<\alpha$ during the second mode, the population decrease is fixed. The function $f d v$ in the second mode is replaced with a constant $c_{2}$.

The system of equations for the analytical model is similar to system (5.1). The simplified version of the model differs by the following summands. The vegetation alienation $D_{V}=V \cdot c_{2}$ during the first mode. The reindeer death $M_{R}=R \cdot b_{2} \quad(V \geq \alpha)$ during the first mode but $M_{R}=R \cdot b_{3} \quad(V<\alpha)$ during the second mode.

\subsection{Analytical Solution}

The food is not available.

$$
\begin{gathered}
R(t)=C_{1}^{*} e^{-b_{3} t} \\
\frac{V(t)}{\left(a_{1}-a_{2}-c_{2}\right)-a_{1} V(t) / \mathrm{V}_{\max }}=E e^{\left(a_{1}-a_{2}-c_{2}\right) t} .
\end{gathered}
$$

There is enough food. 


$$
\begin{gathered}
R(t)=C_{2}^{*} e^{\left(-b_{2}+k p b r b_{1}\right) t}, \\
V(t)=e^{\left(a_{1}-a_{2}\right) t / 2}\left[\begin{array}{c}
\left.C_{1} J_{v}\left(2 b_{2}^{-1} \sqrt{\frac{a_{1} b_{1} C_{2}^{*}}{V_{\max }} e^{b_{2} t / 2}}\right)+\right] \\
\left.+C_{2} Y_{v}\left(2 b_{2}^{-1} \sqrt{\frac{a_{1} b_{1} C_{2}^{*}}{V_{\max }}} e^{b_{2} t / 2}\right)\right] \\
v=\frac{a_{2}}{b_{2}},
\end{array}\right.
\end{gathered}
$$

where $C_{1}^{*}, E, C_{2}^{*}, C_{1}, C_{2}$ are arbitrary constants, they are derived from initial conditions; $J_{v}$ and $Y_{v}$ are the Bessel functions (see [17]).
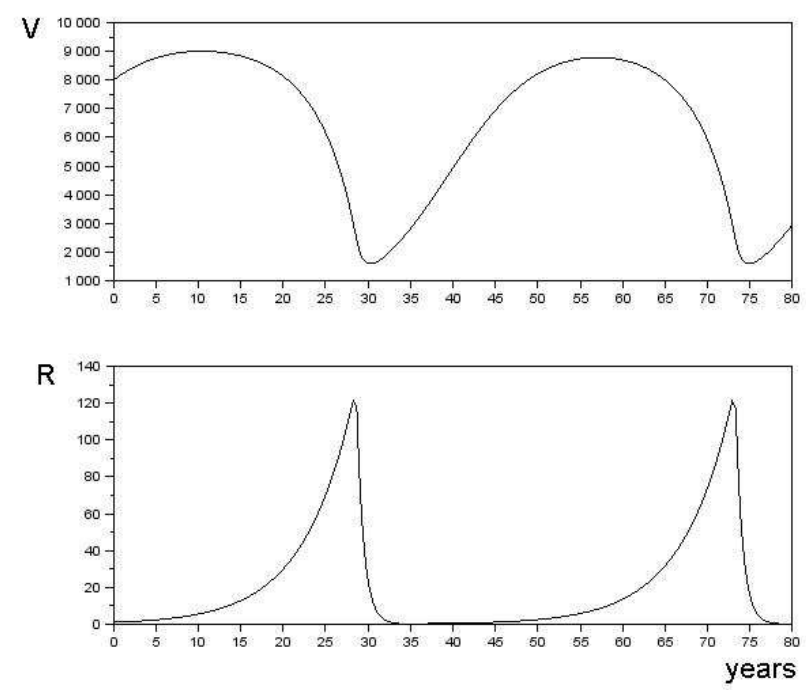

Figure 9. The results of the computational experiments with "vegetation-reindeer" model.

\section{Conclusion}

Simulation modeling in an ecologo-biological field is the art of using computer technology in an interdisciplinary process of creating mathematical models under incomplete and always distorted data of various nature about the properties of the object under study (see [8]). It is the art of compromise between ecological and mathematical requirements.

The search for such successful combinations is based on the idea of the "ecological constructor", i.e., the algorithmic structure of the model that lets one relatively easily modify it. The implementation of this idea is based on Forrester's system dynamics and the Volterra-Kosticyn hypothesis on the possibility to use systems of ordinary differential equations to describe ecological objects (see [5]).

However purely simulation techniques are hard pressed to get a satisfactory description of the mechanisms of the phenomenon under study. A combination of analytic and simulation approaches considering the sets of interrelated models presents an attractive option. The search for ways to implement such combinations has led to the creation of the complex studies method.

The simplified models admitting parametric studies have completely changed the possibilities and potential of the modeling. This is both a tool for tuning the original simulation model in corresponding dynamic modes and a way to generate the hypotheses regarding the leading mechanisms of the phenomenon under consideration.

Our complex approach shows how we can use the computer not only to produce the corollaries of known facts or to input a huge number of parameters but also to simplify the model and generate the hypotheses regarding the mechanisms of the phenomenon under study. Using this approach to model tundra populations and communities we have implemented the idea of simulation technology's efficiency to justify simplified equations admitting the parametric studies. We have created a special class of models such that it takes into account both seasonality (see [14]) and difference equations (see [10]). Our previous modeling experience has let us move to another level of description namely individual-oriented modeling (see [4, 13]). The development of adequate mathematical models for various biological processes is necessary to form the framework of theoretical biology. Besides, under increasing global anthropogenic influences the model approach is virtually the only way to preserve an integral concept of biosphere objects being destroyed.

\section{References}

[1] J. Forrester, Word Dynamics, Massachusetts Wright - Allen Press Inc., Cambridge, 1971.

[2] C.J. Topping, T. Dalkvist, and V. Grimm, "Post-hoc pattern-oriented testing and tuning of an existing large model: lessons from the field vole", Plos One, vol. 7, no. 9, Article Number: e45872, 2012.

[3] V. N. Glushkov and D. A. Sarancha, "A complex mathematical modeling method for biological objects. Modeling the tundra community", Automation and Remote Control, vol. 74, no. 2, pp. 240-251, 2013.

[4] S.N. Boranbayev, D.A. Sarancha, R. Taberkhan, and R.V. Trashcheev, "Applying of combined methods for initiation of mathematical modeling of biogeocenosis in the different region of Kazakhstan (individual-oriented models)", Bulletin of Gumilyov's Eurasian National University, Special Issue, pp.133-142, 2012.

[5] D.A. Sarancha, O.P. Lyulyakin, and R.V. Trashcheev, "Interaction of simulation and analytic methods in modeling of ecological and biological objects", Russian Journal of Numerical Analysis and Mathematical Modeling, vol. 27, no. 5, pp. 479-492, 2012.

[6] F. B. Chernyavskyi and A. N. Lazutkin, The Cycles Lemmings and Voles in the North, Magadan: Russian Academy of Sciences, Far East Branch, North-East Scientific Center, Institute of Biological Problems in the North, 2004. 
[7] D.A. Sarancha, Kolichestvennye metody v ekologii. Biofizicheskie aspekty i matematicheskoe modelirovanie (Quantitative Methods in Ecology: Biophysical Aspects and Mathematical Modeling), Moscow: Mosk. Fiz.-Tekh. Inst., 1997.

[8] J. Murray, Mathematical Biology, vol.1, Springer-Verlag, New York, 2007.

[9] J. J. Nieto, M. J. Pacifico, and J. L. Vieitez, "Long-term and short-term dynamics of microtus epiroticus: a Yoccoz-Birkeland model", Siam Journal on Applied Dynamical Systems, vol. 11, no. 4, pp. 1499-1532, 2012

[10] E. V. Nedostupov, D. A. Sarancha, E. N. Chigerev, and Yu. S. Yurezanskaya, "Some properties of one-dimensional unimodal mappings", Doklady Mathematics, vol. 81, no. 1, pp. 16-21, 2010.

[11] V.A. Orlov, D.A. Sarancha, and O.A. Shelepova, "Mathematical-model of the numerical dynamics of lemmings (Lemmus, Dicrostonyx) and its application for describing the populations of West Taimyr", Soviet Journal of Ecology, vol. 17, no. 2, pp.97-104, 1986.
[12] F.A. Pitelka and G.O. Batzli, "Population cycle of lemmings near Barrow, Alaska: a history review", Acta Theriologica, vol. 52, no. 3, pp. 323-336, 2007.

[13] V. D. Perminov and D. A. Sarancha, "On some approach to solving population ecology problems", Matem. Mod., vol. 15, no. 11, pp. 121-128, 2003.

[14] A.I. Lobanov, D.A. Sarancha, and T.K. Starozhilova, "Seasonality in the Lotka-Volterra model", Biofizika, vol. 47, no. 2, pp. 325-330, 2002.

[15] V.N. Lopatin and B.D. Abaturov, "Mathematical Modeling of Trophically Dependent Cycle of Reindeer (Rangifer Tarandus) Population", Zoologichesky Zhurnal, vol. 79, no. 4, pp. 452-460, 2000.

[16] B.D. Abaturov, "On the mechanisms of natural regulation of the relationship between herbivorous mammals and vegetation”, Zoologichesky Zhurnal, 1975, vol. 54, no. 5, pp. 342-351, 1975.

[17] A. D. Polyanin and V. F. Zaitsev, Handbook of Exact Solutions for Ordinary Differential Equations, 2nd ed., Chapman \& Hall/CPC, Boca Raton, 2003. 